\title{
El diario pedagógico como herramienta para la investigación*
}

\author{
The daily teaching and research tool \\ Alexandra Yulieth Monsalve Fernández** \\ Eliana María Pérez Roldán***
}

RECIBIDO: 10 DE ABRIL DE 2012 • ACEPTADO: 18 DE OCTUBRE DE 2012

Resumen. El diario pedagógico es considerado como una herramienta de gran utilidad para los maestros, no sólo como posibilidad de escritura ni como narración anecdótica de lo que sucede en la clase, sino también como elemento para la investigación. Por tanto, éste no debe concentrarse solamente en los hechos, sino también desde su estructura permitir el abordaje de experiencias significativas, tanto para el maestro como para sus estudiantes.

Mediante la búsqueda de fuentes de información sobre el tema, las investigadoras consolidan una propuesta desde la cual se valida el diario pedagógico como herramienta para la investigación con base en experiencias ya vividas y escritas en otros contextos educativos.

* Artículo de reflexión correspondiente al proyecto titulado: «Identificación y análisis de buenas prácticas pedagógicas en los diarios pedagógicos del Cibercolegio UCN». Investigación adscrita al grupo Cibereducación de la Fundación Universitaria Católica del Norte, en la línea de investigación en Educación Virtual.

** Normalista superior con énfasis en Lengua Castellana, estudiante de Licenciatura en Educación básica con énfasis en Lengua Castellana, Universidad de Antioquia. Facilitadora virtual de Lengua Castellana, Cibercolegio Universidad Católica del Norte (UCN), Colombia. E-mail: aymonsalvef@ucn.edu.co

*** Psicóloga y estudiante de Especialización en Entorno Virtuales de Aprendizaje. Docente de Psicología e integrante del área de Acompañamiento y Capacitación a Facilitadores (docentes) en la Fundación Universitaria Católica del Norte (UCN), Colombia. E-mail: emperez@ucn.edu.co 
Finalmente, se presenta una propuesta para hacer uso de éste dentro de los ambientes virtuales de aprendizaje como fuente de información para enriquecer el modelo de educación virtual.

Palabras clave. Diario pedagógico, investigación en el aula, práctica docente, ambientes virtuales, aprendizaje.

\begin{abstract}
The daily diary is considered a useful tool for teachers, not simply as writing or as anecdotal account of what happens in the classroom, but also as an element in the investigation. Therefore, the newspaper should not focus only on facts but also from its structure to allow the approach of meaningful experiences for both the teacher and students. By searching for sources of information on the subject of a proposed consolidated research from which validates the daily teaching and research tool based on experiences lived and written in other educational contexts. Finally, we present the daily dairy in virtual learning environments as a sour.
\end{abstract}

Keywords. Pedagogical diary, classroom research, reflection on teaching practice, virtual environment, learning.

\title{
Introducción
}

La escritura es una de las formas de comunicación más antiguas y ha permitido preservar legados de todas las épocas, es por ello que se convierte en una práctica habitual de la humanidad, más aún, para los maestros y maestras que renuevan constantemente sus prácticas de acuerdo con las necesidades que surgen en el quehacer cotidiano. Al respecto, Vásquez (2008) sustenta que la escritura se convierte en un material óptimo para el ejercicio de la docencia y, más que ello, como herramienta para la investigación; por ejemplo con el diario de campo, en el cual se entienden dos líneas de sentido, una primera basada en la narración y una segunda regida por la reflexión y la construcción de textos con carácter investigativo. Sin embargo, es común encontrar que en gran parte de los casos no se escribe y quienes lo hacen generalmente no hacen uso de ello como fuente de análisis de su desempeño.

En este sentido, se ha encontrado que en el Cibercolegio Universidad Católica del Norte (en adelante UCN), desde el año 2008 se vienen rea- 
lizando diarios pedagógicos a partir de las experiencias significativas de los facilitadores virtuales, sin hacer un proceso de análisis de este valioso material. Cabe preguntarse entonces de qué manera se puede hacer de los diarios pedagógicos un proceso que trascienda la escritura y genere nuevas posibilidades de trabajo, de investigación, de reflexión, entre otras.

Para comprender mejor el contexto, es propio precisar algunos términos. El Cibercolegio UCN es una institución de educación básica y media que utiliza el modelo de educación virtual y para esta institución, los maestros y maestras se denominan facilitadores. Así mismo, el diario pedagógico es un ejercicio de escritura que realizan los facilitadores acerca de su práctica docente, en donde van registrando algunas reflexiones sobre el día a día de su labor y experiencias que ellos consideran como significativas en el proceso de enseñanza aprendizaje.

En el Cibercolegio UCN se disponen los contenidos desde una plataforma virtual, en la cual se consignan materiales de estudio, que sirven de base para el desarrollo de actividades por parte de los estudiantes. Los facilitadores virtuales tienen contacto con los estudiantes por medio de herramientas sincrónicas, tales como: messenger, skype y el aula virtual Livemeeting durante 6 horas al día; y asincrónicas, como el correo, el espacio para envío de tareas y los foros. Con base en este contacto se desarrollan relaciones alumno-maestro mediadas por la virtualidad que a su vez son la base para la escritura del diario pedagógico, el cual tiene un carácter similar al diario escrito manualmente, pero se almacena en blogs u otros espacios institucionales.

El diario pedagógico se concibe como un texto escrito que, como ya se ha dicho, registra experiencias, sin embargo, adquiere un sentido de carácter más epistemológico que narrativo, en la medida: en que no se limita a la narración de anécdotas, sino que éstas tienen un sustento pedagógico originado en los resultados obtenidos por los facilitadores en determinado momento, los cuales dan lugar a prácticas pedagógicas que se deben tener en cuenta como parte de la cualificación del proceso educativo.

Ello ha dado origen al interés por acercarse a dichas prácticas, a partir de los textos escritos por los facilitadores virtuales dentro de sus diarios. Ahora bien, este artículo surge como producto inicial de la investigación Identificación y análisis de buenas prácticas pedagógicas en los diarios 
pedagógicos del Cibercolegio UCN. Se pretende entonces, exponer si efectivamente es posible utilizar este material escrito para otros propósitos que trasciendan la misma escritura, que de por sí, es ya una práctica valiosa y significativa.

En el marco de la investigación, la pertinencia del artículo está en confirmar si es posible o no encontrar diferentes aplicaciones y usos de los diarios pedagógicos, lo cual daría más relevancia al proceso investigativo en desarrollo, teniendo en cuenta que desde la misma investigación y desde las necesidades de la institución en la que se llevan a cabo, ya se tienen detectados los procesos que se beneficiarían de los resultados que se obtengan, principalmente en lo referente al acompañamiento a facilitadores que desde allí se realiza.

Dicho acompañamiento se basa en orientar a los facilitadores en el modelo de educación virtual, aproximándolos al uso pedagógico y didáctico de las herramientas tecnológicas que se utilizan en función de la consolidación de dicho modelo pedagógico.

\section{Método}

El proceso de investigación que se ha venido adelantado es de corte cualitativo y tiene un carácter descriptivo; este artículo se ha desarrollado como parte de un primer momento investigativo en el cual se construyó un estado del arte con base en la revisión del tema objeto: los diarios pedagógicos. De acuerdo con Best (1982, p. 91), la investigación descriptiva refiere minuciosamente y refiere lo que es. Está relacionada con condiciones o conexiones existentes; prácticas que prevalecen, opiniones, puntos de vista o actitudes que se mantienen; procesos en marcha, efectos que se tienen o tendencias que se desarrollan.

Se han consultado diversas fuentes de información teniendo en cuenta los intereses propios del proceso de investigación, para lo cual se hizo una clasificación del material encontrado, con miras a rescatar aquellas experiencias que dan lugar a propuestas conceptuales con base en el abordaje del diario pedagógico como recurso para analizar, problematizar y resignificar el aula de clase.

Los tópicos usados para la búsqueda de información relacionada fueron: diario de campo, diario pedagógico, diario, buena práctica; encontrando 
que, si bien es posible hallar trabajos de campo con base en los diarios pedagógicos como fuentes para adquirir información acerca del proceso que se lleva a cabo en la clase, no se han desarrollado investigaciones con miras a identificar las buenas prácticas que se reflejan en los diarios escritos por los maestros con el fin de fortalecer la práctica como tal desde diversas miradas.

Para las búsquedas se utilizaron bases de datos, tales como Redalyc y Dialnet, además de la biblioteca Central y el Centro de Documentación de la Facultad de Educación de la Universidad de Antioquia. En estos espacios se encontraron artículos de reflexión acerca de los diarios pedagógicos y/o de campo, encaminados hacia la comprensión del diario como fuente de saber epistemológico y de reconocimiento de la clase.

A medida que se fueron encontrando los textos, se registraron en fichas de lectura las fuentes que se consideraron pertinentes para el trabajo investigativo, dejando de lado las que no aportaron información relevante. Posteriormente, las fichas de lectura se consultaron progresivamente para articular el material a la formulación del proyecto y construcción del artículo. La base para la elaboración de las fichas de lectura consistió en la toma de información sobre la ubicación y referencia del texto, además de un resumen para orientar la utilidad del mismo dentro del proceso global.

Con base en el proceso de búsqueda de la información se han dado unos primeros hallazgos a nivel conceptual, que finalmente constituyen la base de análisis para los diarios pedagógicos escritos por los facilitadores virtuales del Cibercolegio UCN. A continuación se presenta información al respecto.

\section{Estructura del diario pedagógico}

Como ya se ha dicho, el diario pedagógico es un sistema de registros usado para hacer referencia a diversas situaciones que ocurren dentro de la clase; en este se hace énfasis tanto en lo disciplinar como en lo práctico.

El diario pedagógico se entiende como una fuente que usa el docente investigador para reconocer aspectos que se dan dentro de la clase y que no se identifican en el momento en que ocurren, sino que surgen como parte del análisis de los registros y después de la relectura mencionada 
por Acero (s.f.). El carácter secuencial permite identificar situaciones o acciones que se repiten y, por tanto, se convierten en prácticas usuales del docente, en este caso del facilitador virtual, quien debe entender el diario como una fuente para realizar exploraciones sistemáticas dentro de su labor educativa.

Dentro de las búsquedas realizadas se han encontrado diversas formas de hacer el diario pedagógico, entre ellas se resaltan la de Acero (s.f.) y la de Porlán \& Martín (1991), quienes desde sus intereses dan algunas recomendaciones acerca de la manera en que debe redactarse el diario.

Para Acero «el diario de campo es el instrumento que favorece la reflexión sobre la praxis, llevando a la toma de decisiones acerca del proceso de evolución y la relectura de los referentes, acciones estas, normales en un docente investigador, agente mediador entre la teoría y la práctica educativa» (sf. p.14): por lo tanto, la elaboración del diario pedagógico supone el desarrollo de la capacidad reflexiva mediante la cual se identifican y solucionan situaciones que afectan la clase, del mismo modo en que se potencian otras que le dan valor a las prácticas generadas, tales como las fortalezas halladas en cuanto al acompañamiento a los estudiantes, la comunicación dentro de la institución y el uso de estrategias metodológicas que han dado buenos resultados.

Para Acero (s.f) el diario debe iniciar con la lectura de la realidad, en la cual se sistematice todo lo que ocurre en el momento formativo, tanto dentro como fuera del aula de clase. En el diario se deben identificar las expectativas y saberes propios con respecto a los de los estudiantes, los roles de ambos y los momentos significativos, presentando desde un punto de vista personal situaciones del docente, el alumno y la comunidad que pueden ser clasificadas posteriormente para procesos de investigación.

Porlán \& Martín (1991) menciona que el diario se debe iniciar desde lo general para llegar hasta lo concreto; en el primer nivel se explicitan situaciones de origen anecdótico que dan lugar a la construcción más profunda en la cual se describen las dinámicas propias dentro del aula de clase y el sistema escolar.

En un segundo nivel el diario se encarga de reflejar problemas y concepciones que atraviesan la dinámica de la clase, para establecer las 
interpretaciones que se puedan hacer al respecto. Continuando con la propuesta de Porlán \& Martín (1991) es posible entonces afirmar que, si bien inicialmente se pueden presentar dificultades en cuanto a la subjetividad de las interpretaciones, con el paso del tiempo se podrán superar, puesto que se convierte en un análisis sistemático.

El diario pedagógico se puede constituir también en una herramienta mediante la cual el facilitador almacena estrategias formativas con resultados exitosos, con el fin de dar continuidad a las mismas.

En el caso del Cibercolegio UCN, el diario pedagógico debe partir de situaciones cotidianas que enmarcan el proceso educativo, mediante la narración de buenas prácticas realizadas por los facilitadores virtuales; dando lugar a experiencias de aprendizaje significativo, tanto para los facilitadores como para los estudiantes, por lo cual, en un primer momento se hace una narración anecdótica de las experiencias que han sido importantes durante el tiempo de realización que cubre el diario, para pasar a una reflexión desde la cual se construyen saberes de gran utilidad en la formación de estudiantes virtuales.

Armijo (2004), citado por Jerí (2008), señala que las buenas prácticas son experiencias con buenos resultados y que se orientan a soluciones concretas y efectivas que posibilitan una mejora en el desempeño. Adicionalmente, Jerí (2008) indica un primer rasgo de las buenas prácticas, que se sintetiza en la expresión: una buena práctica es una experiencia de carácter innovador que permite solucionar un problema a través de una mejora en el proceso. Un segundo rasgo, es el carácter de una buena práctica, ya que ésta tiene una base cognitiva unida a un actuar, por lo tanto, está ligada a un carácter procedimental.

\section{Usos del diario}

Después de haber realizado una revisión de la literatura que se encuentra con respecto al tema de interés, es posible afirmar que el diario pedagógico es una herramienta de gran utilidad para el docente, como espacio de construcción y reconocimiento de saberes, Vásquez (2002) hace un análisis del diario de campo como un referente fundamental para la clasificación, codificación y categorización de la información, dentro de un proceso de investigación etnográfica, por lo cual el diario se convierte en un objeto de estudio que aporta datos, permitiendo registrar 
los hallazgos de cada sesión. Por lo cual, a su vez puede convertirse en un modelo de evaluación progresiva desde las competencias que dentro de él se enuncian.

Si bien una de las principales utilidades del diario pedagógico es la de cumplir con un espacio de registro de información y sucesos importantes para la escuela, esta primera medida debe trascenderse con miras a hacer del mismo un sistema mediante el cual se evalúen y reconstruyan las prácticas de manera que se reflexione sobre lo que narra el facilitador o docente con miras al reconocimiento de buenas prácticas que pueden ser compartidas como experiencias exitosas para la enseñanza.

\section{Experiencias de uso del diario}

Durante el proceso de investigación y rastreo de antecedentes, se encontró que existen algunas experiencias institucionales que dan cuenta de las posibilidades de uso para los diarios pedagógicos.

Entre estas experiencias se puede encontrar la utilización de los diarios pedagógicos como fuente de consulta, observación y análisis para el proceso de autoevaluación institucional de la Escuela Normal Superior Pedro Justo Berrio (2007). Dicha institución, que se encuentra en el municipio de Santa Rosa de Osos (Antioquia) y se dedica a la educación básica y media desde hace cien años, en donde durante el año 2007, se llevó a cabo un proceso de autoevaluación institucional, en el que fueron utilizados los diarios pedagógicos de los docentes como el insumo principal para identificar el cumplimiento o la evidencia de algunos indicadores planteados para medir o evaluar el estado de la institución frente a ellos y frente a los requisitos correspondientes a tal autoevaluación, es de anotar además que dicho proceso de análisis del diario es fundamental en el futuro institucional, en vista de que es en su esencia una institución formadora de maestros.

Estos indicadores se formularon para evaluar los siguientes componentes:

- Práctica pedagógico didáctica.

- Actores (directivos, maestros, alumnos, padres de familia).

- Organización y gestión.

- Cultura escolar (local, regional, nacional, mundial). 
Dichos componentes se analizaron en tres dimensiones: sustento pedagógico, estructura académica e investigación, para cubrir en todo su alcance, la estructura necesaria para el funcionamiento de la institución de acuerdo con los estándares de calidad que aplican para ella. En este sentido, al revisar los diarios y la escritura de los profesores sobre las experiencias vividas día a día y sus propias reflexiones frente a las mismas, se sondeó si existían tales evidencias o si, por el contrario, los componentes no estaban presentes en su reflexión, motivo que daría paso a la formulación de estrategias para potencializar el componente en la institución.

A partir de la experiencia de observación de los diarios pedagógicos, en la Escuela Normal se logró detectar el nivel de cumplimiento de los indicadores propuestos en las prácticas docentes, de manera que se lograra establecer qué aspectos correspondientes a los criterios de calidad deberían ser fortalecidos en la institución. De aquí entonces, que sea posible que los criterios de los diarios pedagógicos se puedan trascender y llevar a prácticas significativas en el entorno escolar que impacten el qué hacer de los profesores y evaluar procesos pedagógicos a nivel institucional.

Adicionalmente, cada indicador formulado constituye un ejemplo de opciones, posibilidades o como pueda llamarse, para transformar en productos de impacto pedagógico y, en el caso de la educación virtual, sería bastante enriquecedor asumir este riesgo y dar pasos en esa vía. Lo anterior se complementa con lo afirmado por Salinas (2000, p. 6), quien propone que a pesar de su carácter de registro, el diario pedagógico no puede confundirse con un instrumento de control o de repetición de acciones. En éste el maestro debe reflejar sus vacíos formativos, sus anécdotas pero a raíz de ellas siempre hacer una reflexión o teorización sobre el diario vivir de la institución, con miras hacia la investigación sobre la práctica y como elemento para identificar los problemas de la escuela.

Otra propuesta, es presentada por Vain (2003, p. 58) quien indica que se registran experiencias de uso del diario, en las cuales se aborda como estrategia para autoevaluar la práctica desde un punto de vista epistemológico; de este modo se plantea una ruptura entre la escuela tradicional y la escuela contemporánea estrechamente ligada a la escritura como ejercicio docente. Al respecto, se destaca que el diario académico 
permite establecer una concepción epistemológica de la educación, identificando con facilidad los problemas y las posibles soluciones; en este sentido es adecuado retomar los diarios escritos por estudiantes, con el fin de integrar los conceptos científicos con los modos en que ellos los interpretan la realidad.

En el caso del Cibercolegio UCN, en una entrevista realizada a Omar Ruiz, el 18 de mayo de 2011, manifestó que en su época como coordinador se tenía un link en internet, en formato wiki, el cual permitía que los facilitadores de manera permanente estuvieran alimentando su diario pedagógico, además de que posibilitaba la revisión del mismo por parte de la coordinación y observaciones al mismo, la idea inicial al plantear la realización de diarios pedagógicos fue la de generar una reflexión sobre la propia práctica docente.

La finalidad de los diarios pedagógicos que se puede percibir en las respuestas del primer coordinador que decidió implementarlo, es la de hacer seguimiento a las acciones pedagógicas significativas y los trabajos desarrollados por los facilitadores virtuales. Para ello el coordinador académico los revisaba y aunque no hay nada escrito al respecto de reflexiones frente al tema, Ruíz (2011), manifestó que tenía como propósito hacer reflexión de la práctica docente, y que, como consecuencia servía para que a nivel personal se hicieran ajustes didácticos, metodológicos y pedagógicos.

\section{Conclusiones}

En suma, se puede encontrar que los usos de los diarios pedagógicos pueden ser tan variados como los intereses de quienes puedan ver en ellos una posibilidad de trascendencia de la escritura, o más bien, de aprovechamiento de la información que allí se puede encontrar para la trasformación y reflexión del proceso de enseñanza - aprendizaje según la visión de los profesores.

En el caso del Cibercolegio UCN, esta trascendencia apuntará al enriquecimiento del modelo de educación virtual que se imparte en la institución, así como en el fortalecimiento del repertorio de las estrategias pedagógico - didácticas que suelen utilizar los facilitadores al interior de sus cursos, y que en muchos casos por estar en un contexto virtual, su difusión entre los colegas puede ser menor. 
Por otro lado, tendremos la posibilidad de aproximarnos a la concepción de educación virtual en la educación básica y media sobre la que los facilitadores hacen su práctica pedagógica, esto es, una manera de encontrar en la práctica una modalidad de educación desde la perspectiva de quien la lleva a cabo.

Como se ha logrado evidenciar, el diario pedagógico es una herramienta de gran valor para el docente, siempre y cuando se use de una forma completa que avance más allá de la escritura anecdótica; por tanto, un docente comprometido con su labor desde el carácter investigativo que puede darle a la misma, debe asumir el diario pedagógico como material de análisis sobre su práctica, y por supuesto, como elemento para la mejora continua de la misma.

Para el proceso de investigación que se adelanta se tiene como una de las metas fundamentales, la identificación de registros escritos en los diarios pedagógicos que den lugar a construcciones académicas en las cuales se establezcan pautas de acción basadas en las buenas prácticas, teniendo como base para ello el modelo educativo institucional y pensando la forma en que desde los diarios pedagógicos se refleja la identidad del facilitador y el estudiante frente al proceso.

\section{Referencias}

Acero, E. (s.f.). El diario de campo: Medio de investigación del docente. En Actualidad Educativa, 3 (13). 13.

Best, W. (1982). Cómo investigar en educación. Madrid: Ediciones Morata.

Escuela Normal Superior Pedro Justo Berrío (2007). Autoevaluación Institucional. 35-48.

Jerí, D. (2008). Buenas prácticas en el ámbito educativo y su orientación a la gestión del conocimiento. En Educación. 17 (33), 28-48.

Porlán, R. \& Martín, J. (1991). El diario del profesor, un recurso para la investigación en el aula. Sevilla: Diada.

Ruiz, O. (2011). Entrevista personal realizada el 18 de mayo de 2011.

Salinas, M. (2000). El diario pedagógico. En Revista la gaceta didáctica, 3,15 . 
Vain, P. (2003) El diario académico: una estrategia para la formación de docentes reflexivos. En Revista Perfiles Educativos, 100, 56-68.

Vásquez, F. (2002). El diario de campo una herramienta para investigar en preescolar y primaria. En Serie formación de maestros Bogotá D.C, Proyecto de restructuración de escuelas normales. 111.

(2008). La escritura y su utilidad en la docencia. En Revista Actualidades Pedagógicas, (051), 101-114. Recuperado de http:// redalyc.uaemex.mx/pdf/956/95605107.pdf 